\title{
AUTOMATED EXTRACTION OF BUILDINGS FROM AERIAL LIDAR POINT CLOUDS AND DIGITAL IMAGING DATASETS
}

\author{
Ali Jamali ${ }^{1 *}$, Pankaj Kumar² and Alias Abdul Rahman ${ }^{3}$ \\ ${ }^{1}$ Faculty of Surveying Engineering, Apadana Institute of Higher Education, Shiraz, Iran- ali.jamali.65@ gmail.com \\ ${ }^{2}$ School of Natural and Built Environment, University of South Australia, Adelaide, Australia- pankaj.kumar2@unisa.edu.au \\ ${ }^{3}$ Universiti Teknologi Malaysia (UTM), Faculty of Built Environment and Surveying- alias@utm.my
}

KEYWORDS: LiDAR; digital imaging; buildings extraction; OBIA; NGBDI

\begin{abstract}
:
To acquire 3D geospatial information, LiDAR technology provides the rapid, continuous and cost-effective capability. In this paper, two automated approaches for extracting building features from the integrated aerial LiDAR point cloud and digital imaging datasets are proposed. The assumption of the two approaches is that the LiDAR data can be used to distinguish between high- and low-rise objects while the multispectral dataset can be used to filter out vegetation from the data. Object-based image analysis techniques are applied to the extracted building objects. The two automated buildings extraction approaches are tested on a fusion of aerial LiDAR point cloud and digital imaging datasets of Istanbul city. The object-based automated technique presents better results compared to the threshold-based technique for extraction of building objects in term of visual interpretation.
\end{abstract}

\section{INTRODUCTION}

Many applications such as cadastral mapping, 3D city modeling, infrastructure mapping, and urban growth analysis require 3D extraction of buildings. Additionally, accurate and up-to-date information about the location and dimension of building features in an urban environment provides crucial input for the fire-safety analysis and managing other hazards including 3D flood simulation. Traditionally, from closerange and satellite images, building boundaries are extracted based on manual or semi-automated reconstruction. These processes are costly, time-consuming and limited to 2D reconstruction of building objects. The lack of automated methods can be defined as problems in finding appropriate information from the data and the complexity of the scene (Elberink, 2008). Advances in geospatial data acquisition techniques have transformed the concept of $2 \mathrm{D}$ building modeling to $3 \mathrm{D}$.

The automatic reconstruction of urban 3D models has been a research area of photogrammetry for the past two decades (Haala and Kada, 2010). Digital 3D modeling of complex buildings has been a challenge until now with photogrammetry technology (Habib et al., 2010) where advanced photogrammetric based 3D reconstruction methods are fully automated (Musialski et al., 2013). In 3D city modeling, there is an increased demand for 3D point cloud technologies such as LiDAR (light detection and ranging) due to encountered difficulties for interpretation of photogrammetric images, especially for complex buildings.

For automated point cloud segmentation, there are three main techniques including region growing based techniques (Khaloo and Lanttanzi, 2017), model fitting-based techniques (Schnabel et al., 2007) and clustering-based techniques (Biosca and Lerma, 2008). LiDAR enables 3D modeling of the real-world environment by measuring the time of the return of an emitted light pulse (Kumar et al., 2013). Laser scanning systems use this technology to acquire an accurately georeferenced set of highly dense LiDAR point cloud data (Kumar, 2012). A high level of automation during data acquisition is provided by these systems and they have an ability to capture data beneath the tree's canopy.

The applicability of laser scanning systems continues to prove their worth in geospatial mapping due to the rapid, continuous and cost-effective 3D data acquisition capability (Barber et al., 2006). For extracting various features, LiDAR data records a number of attributes including elevation, intensity, pulse width, multiple returns and range information (Kumar et al., 2015). The methods developed for segmenting LiDAR data are mostly based on the identification of planar surfaces and the classification of point cloud data based on its attributes (Vosselman, 2009).

For the past decade, automated extraction of building objects has been a topic of intensive research. Several approaches have been developed for extracting urban building features from LiDAR data over the last decade. For extracting building objects from the integration of airborne LiDAR and digital imaging datasets, a semi-automated technique was developed by Mumtaz et al. (2009). In their approach, the Normalized Digital Surface Model (NDSM) was generated from LiDAR and a Normalized Differential Vegetation Index (NDVI) was developed from the digital image. Both the NDSM and NDVI values were thresholded and then morphological operations were applied to the binary image for extracting building features. However, some of the large vehicles and industrial installations were incorrectly identified as buildings while smaller buildings were missed in their proposed semi-automated method.

A method to extract building features from aerial LiDAR data was proposed by Oda et al. (2004) where the Digital Surface Model (DSM) was segmented and then Hough transformation was applied for extracting building boundaries. Finally, the 3D building model was created by attaching vertical walls from an aerial image to each of the extracted building polygons. 
Pu et al. (2006) developed an approach where LiDAR point cloud was segmented based on the planar surface growing algorithm and then several human pieces of knowledge have driven feature constraints such as size, position, direction, and topology were applied to extract building features. Mancini et al. (2009) developed an automated urban building and road objects extraction method using multi-source aerial LiDAR and multispectral dataset. They involved multi-class supervised pixel classification using an adaptive boosting algorithm to classify buildings, grass, land and tree objects. Finally, filtration and Hough transformation techniques were applied to extract linear road and roundabout features.

Rutzinger et al. (2009) extracted vertical walls from mobile and airborne laser scanning data. To extract planar surfaces from point cloud data, a region growing segmentation technique based on 3D Hough transform was used and then the extracted segments were analyzed based on their inclination, size, and dimension.

Based on the literature, most of the methods developed for extracting buildings require semi-automated or manual intervention. One of the biggest issues in the developed methods are associated misclassification of large vehicles, trees and other features as building objects. There is a need to develop an operational and automated approach for extracting building features. The integration of multispectral digital images with LiDAR data will provide a more efficient and accurate extraction of buildings. The use of LiDAR data provides to distinguish between high- and low-rise objects while multispectral data helps to distinguish vegetations from the building objects.

In this paper, two automated techniques for extraction of building objects from the integration of aerial LiDAR and multispectral digital imaging datasets are developed. Following this Section, in Section 2, the two object-based image analysis technique based on aerial LiDAR and multispectral digital imaging dataset methodologies to extract buildings is presented. Results of the proposed automated techniques are discussed in Section 3. Finally, the accuracy of the results of both proposed techniques is compared and discussed in Section 4.

\section{METHODOLOGY}

\section{1 the Threshold-based automated technique}

Both automated building extraction approaches are tested on aerial LiDAR and multispectral image datasets of Istanbul city which were acquired in October 2012. The multispectral image consisted of three bands including red, blue and green with a ground sampling distance of $0.1 \mathrm{~m}$ and 8-bit radiometric resolution. The point cloud was associated with elevation, intensity, and multiple return attributes. Two proposed methodologies are based on the integration of aerial LiDAR and digital imaging dataset to extract buildings. A workflow of the first automated building extraction approach is shown in Figure 1.

To remove vegetations from the data, digital imaging dataset is used. The available multispectral digital image consisted of blue, green and red bands which represent brightness information of the targets. A low reflectance property of the vegetation in the red band is utilized to suppress them in the data. In the first method, an empirically estimated $\mathrm{T} 1$ threshold value to the red band image is used in order to remove the vegetation area.

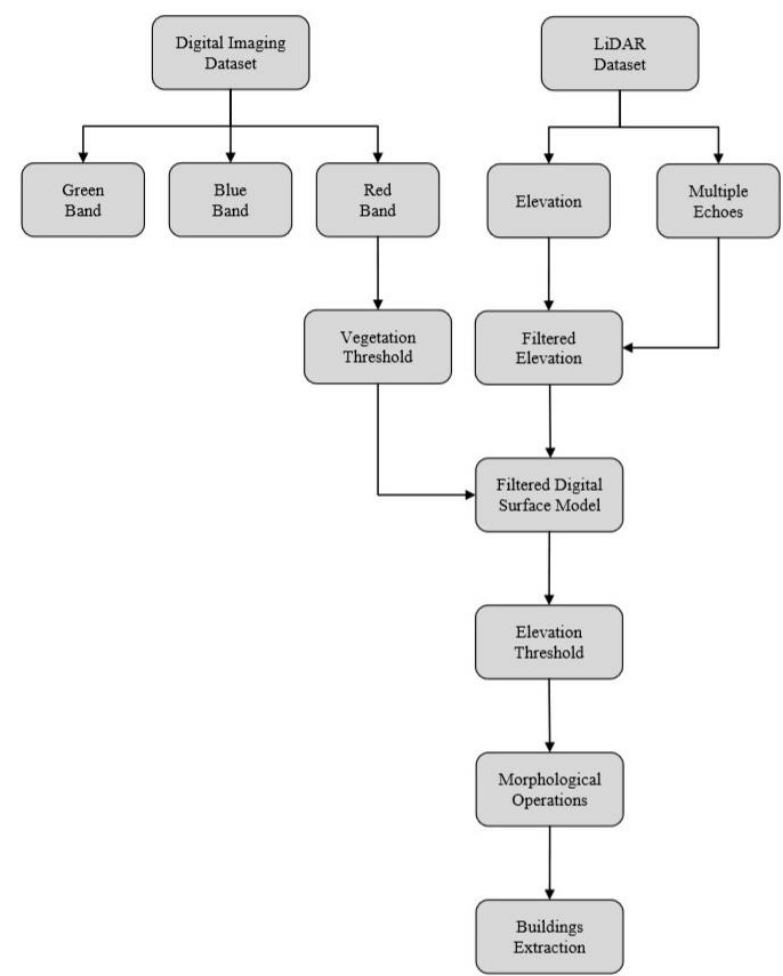

Figure 1. The threshold-based automated buildings extraction approach.

LiDAR data provides multiple return information which refers to multiple returns of return pulses from the targets. Multiple reflected points are filtered out and those points that record a single reflection are retained. These single return reflection points belong to buildings, roads, and other solid objects. The filtered points are used to generate Digital Surface Model (DSM) using the maximum elevation value of points within the cell while natural neighborhood interpolation method is used to estimate values for cells that do not have points within their extent.

The thresholded red band image is used to remove vegetation area from the DSM. An empirically estimated T2 threshold value to the DSM is utilized in order to remove ground-level objects such as roads, parking areas, etc. and retain high rise building features in the data. In order to complete the extracted buildings and remove noise that is introduced through the use of thresholding, the binary morphological operation is used. The thresholded DSM is converted into a binary image and is processed using morphological operations. The morphological opening operation is applied in which the binary image is eroded followed by their dilation while the morphological closing operation is applied by dilating the binary image followed by their erosion. In the dilation operation, a binary matrix element is used to dilate the image pixels and in the erosion operation, a binary matrix element is used to erode the image pixels (Kumar et al., 2014).

Thus, the morphological operations applied to the binary image are able to extract inherent shapes of the building objects and to remove noise. Finally, the contour boundary of each building object is identified and then LiDAR points inside each boundary are estimated to provide 3D generation of extracted building objects. The empirically estimated $\mathrm{T} 1=$ 
130 threshold value was applied to red band image in order to remove vegetation. The DSM was generated from the maximum elevation value of filtered points with $0.1 \mathrm{~m}$ cell size. The empirically estimated $\mathrm{T} 2=45$ threshold value to the DSM is used. The morphological opening and closing operations were applied using a $3 \times 3$ matrix element.

\subsection{The Object-based automated technique}

In this Section, a rule-based object-oriented image analysis technique in eCognition Developer is developed to extract 3D building objects as follow (see Figures 2 and 3):

In this experiment, A bigger data set with more vegetation regions compared to the first experiment is used (see Figure 2a). To calculate the relative elevation which is a subtract of DSM and Digital Elevation Model (DEM), from aerial LiDAR, DSM and DEM are generated. A multi-resolution segmentation algorithm is used to segment the study area where red, blue and green bands have the weight of one and the relative elevation has a weight of 10 . In the segmentation, for the composition of homogeneity criterion, for shape and compactness, the value of 0.3 is selected. The multiresolution segmentation basically minimizes the average heterogeneity of image objects using an iterative algorithm, where objects are grouped until the upper object variance as a threshold is reached.

The variance threshold (scale parameter) is weighted with shape and compactness parameters to minimize the fractal borders of the objects. For vegetation extraction several indices including Red-Green-Blue Vegetation Index (RGBVI, Bendig et al., 2015), Green Leaf Index (GLI, Louhaichi et al., 2001), Visible Atmospherically Resistant Index (VARI, Gitelson et al., 2002), and Normalized Green Red Difference Index (NGRDI, Tucker, 1979) were tested where none of them showed good results. In this study, The Normalized Green Blue Difference Index (NGBDI, see Equation 1 and Figure 2b) is used to extract vegetation areas which show pretty good results compared to the mentioned indices.

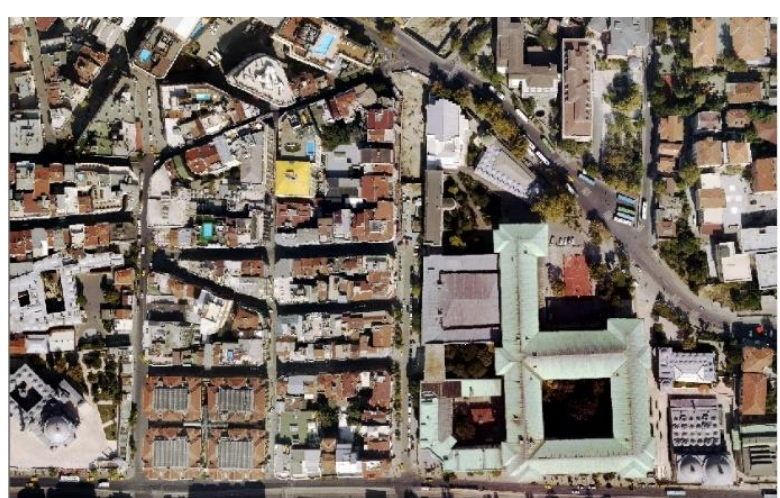

(a)

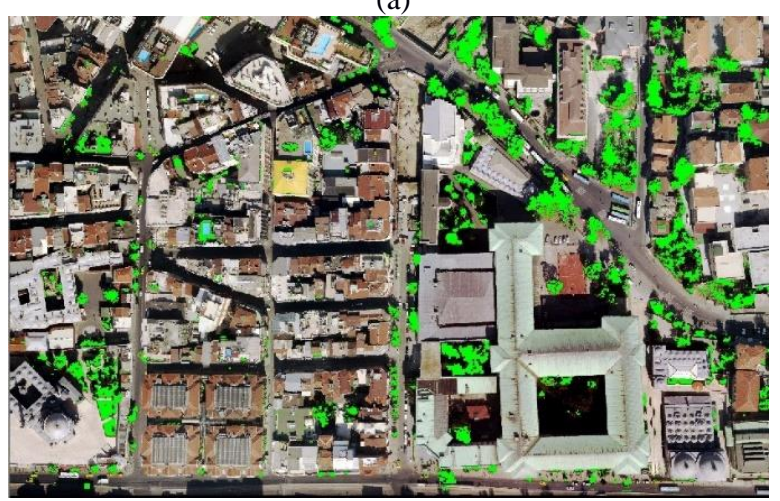

(b)

Figure 2. (a) Input multispectral image and automated extracted (b) Vegetation based on NGBDI.

For vegetation extraction, mean red band with an empirical value of less than 150 , the number of returns from LiDAR data with an empirical value of 0.6 and NGBDI with an empirical value of greater than 0.17 is combined. From the relative elevation, unclassified areas with an elevation of fewer than 3 meters are considered as ground areas where building objects are classified from regions with an elevation of greater than 3 meters.

$$
\frac{(\text { Green }- \text { Blue })}{(\text { Green }+ \text { Blue })}(1)
$$


The International Archives of the Photogrammetry, Remote Sensing and Spatial Information Sciences, Volume XLII-4/W16, 2019 6th International Conference on Geomatics and Geospatial Technology (GGT 2019), 1-3 October 2019, Kuala Lumpur, Malaysia

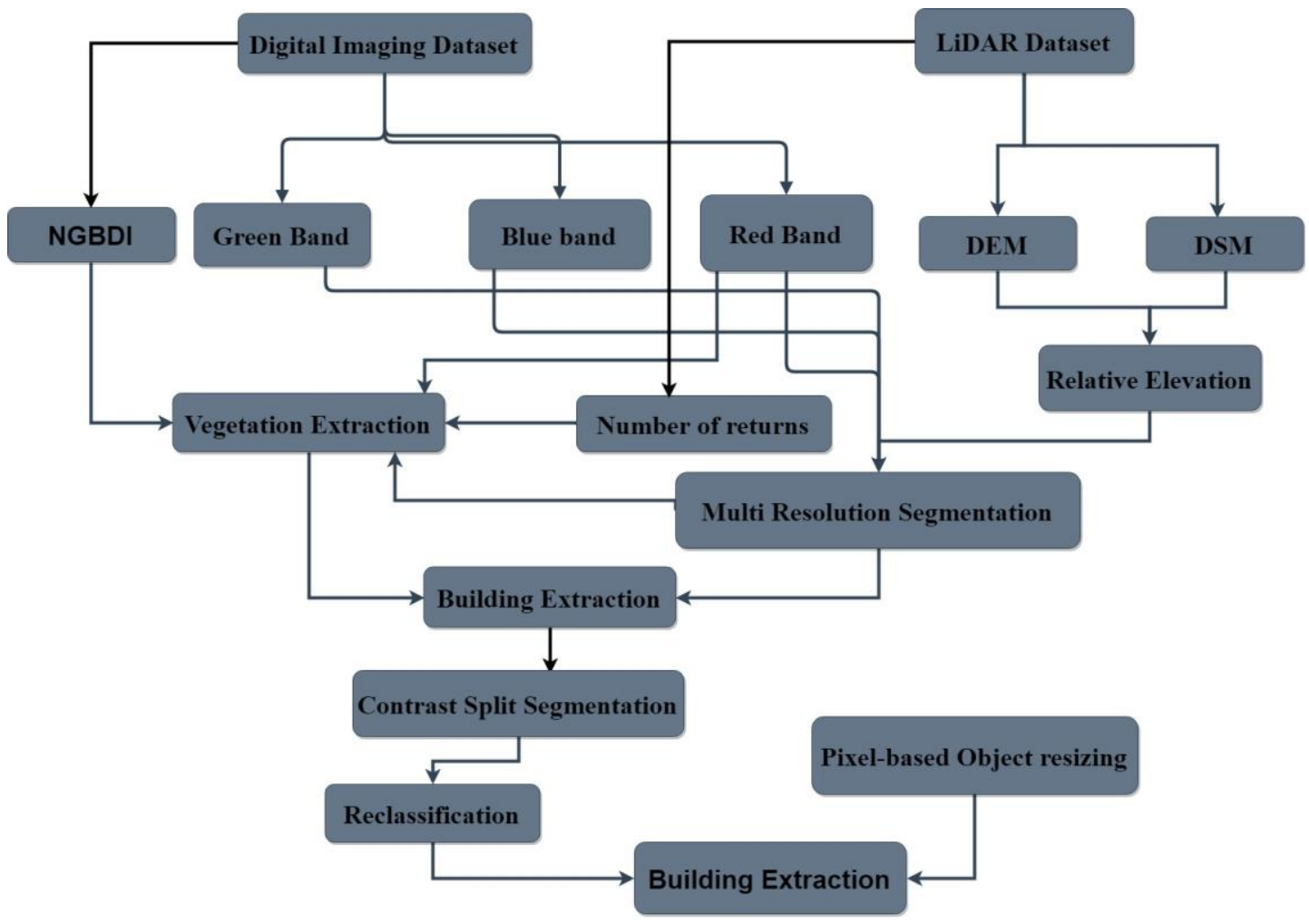

Figure 3. The object-based automated buildings extraction approach.

\section{RESULTS}

\subsection{Results of the threshold-based technique}

The tested multispectral image is shown in Figure 4(a) while the automated extracted 2D and 3D building objects are shown in Figures 4(b) and 5 respectively.
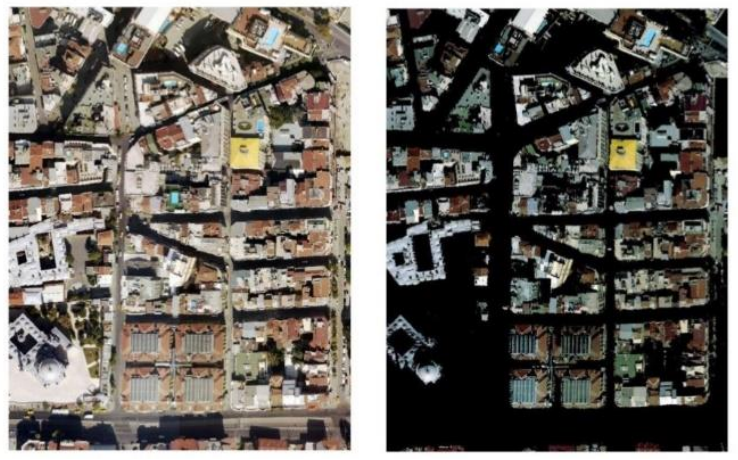

Figure 4. (a) Input multispectral image and automated extracted (b) 2D building objects.

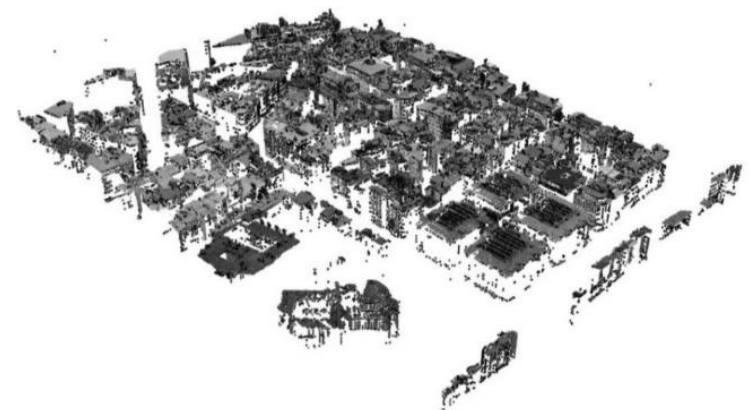

Figure 5. The Automated extracted 3D building objects.
Some of the building objects along the lower-left side of the data were missed while some of the roads were extracted along the middle-right side of the data as false positive as seen in Figure 2(b). Red band in the multispectral image is utilized to remove canopies from the data however, this information was not adequate. The opening and closing morphological operations were applied to complete the shapes of extracted buildings and remove noise. There is a need for their inclusive use in which the dimensions of the extracted objects can be used to remove non-building objects.

\subsection{Results of the object-based technique}

Results of automated building extraction using the objectbased technique is presented in Figures 6 and 7.

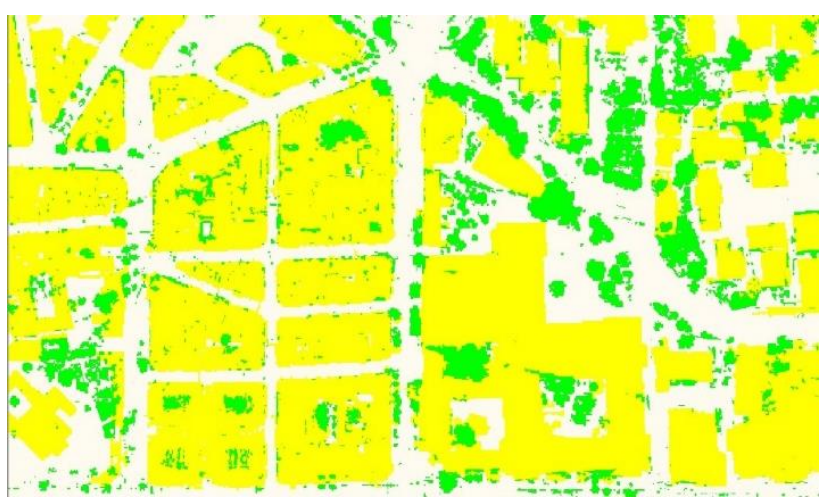

Figure 6. Buildings (yellow), vegetation (green) and ground area (white) classification based on the OBIA technique. 


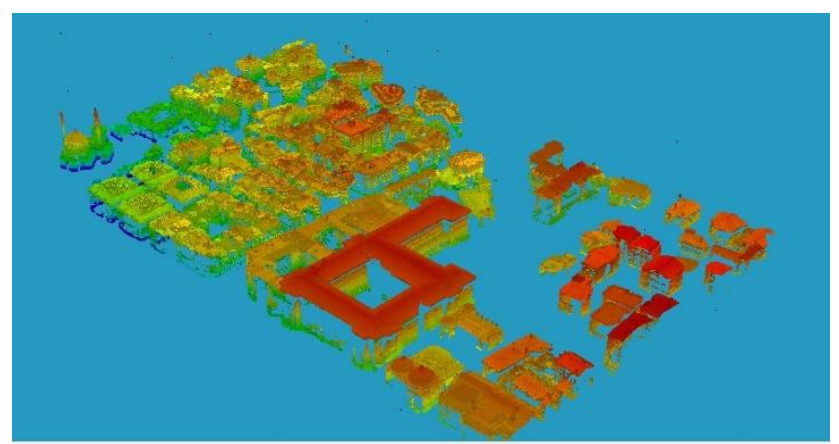

Figure 7. The automated extracted 3D building objects using OBIA technique.

As seen in Figures 6 and 7, although some of the building objects with low elevations are wrongly classified as vegetation areas, results of object-based image analysis technique show good results for building objects identification. In the second automated approach, there are less noise and roads are classified better compared to the first automated approach. In this study, separation of vegetation objects including trees were the biggest issue due to the lack of NIR band. In the second approach, the number of returns raster generated from LiDAR data and NGBDI facilitates better vegetation identification.

\section{CONCLUSIONS}

Two automated approaches were able to successfully extract the building objects from aerial multispectral digital imaging and LiDAR point cloud datasets. The use of both the near infra-red and red bands would provide us to estimate Normalized Differential Vegetation Index (NDVI) which would be more efficient in removing vegetation areas from the data. The use of multiple return attribute in the LiDAR data was further helpful in retaining the points that belong to buildings, roads, and other solid objects.

LiDAR data provides intensity attribute that represents the maximum amplitude of a reflected pulse. Intensity values can be used to differentiate buildings from other terrain objects. The minimum elevation value of points within the cell can be used to generate a Digital Terrain Model (DTM) which can be further used to estimate the Normalized Differential Surface Model (NDSM). NDSM values can be more efficiently used to remove ground-level objects and retain building objects as they represent absolute height values of the terrain objects. This research study presents results for extracting building objects from the integrated aerial LiDAR point cloud and digital imaging datasets where NGBDI is used for vegetation extraction for RGB images.

\section{REFERENCES}

Barber, D., Mills, J. and Smithvoysey, S. (2008). Geometric validation of a ground-based mobile laser scanning system. ISPRS Journal of Photogrammetry and Remote Sensing, 63 (1), pp. 128-141.

Bendig, J., Yu, K., Aasen, H., Bolten, A., Bennertz, S., Broscheit, J., Gnyp, M.L., and Bareth, G., 2015. Combining UAV-based plant height from crop surface models, visible, and near infrared vegetation indices for biomass monitoring in barley. International Journal of Applied Earth Observation and Geoinformation, 39, pp. 79-87.

Biosca, J. M., \& Lerma, J. L. (2008). Unsupervised robust planar segmentation of terrestrial laser scanner point clouds based on fuzzy clustering methods. ISPRS Journal of Photogrammetry and Remote Sensing, 63(1), 84-98.

Elberink, S. O. (2008). Problems in automated building reconstruction based on dense airborne laser scanning data. International Archives of Photogrammetry, Remote Sensing and Spatial Information Sciences, 37 (XXX), pp. 93-98.

Gitelson, A. A., Kaufman, Y. J., Stark, R., \& Rundquist, D. (2002). Novel algorithms for remote estimation of vegetation fraction. Remote sensing of Environment, 80(1), 76-87.

Haala, N., \& Kada, M. (2010). An update on automatic 3D building reconstruction. ISPRS Journal of Photogrammetry and Remote Sensing, 65(6), 570-580.

Habib, A.F., Zhai, \& R., Kim, C. (2010). Generation of complex polyhedral building models by integrating stereoaerial imagery and LiDAR data. Photogrammetric Engineering \& Remote Sensing, 76(5), 609-623.

Khaloo, A., \& Lattanzi, D. (2017). Robust normal estimation and region growing segmentation of infrastructure 3D point cloud models. Advanced Engineering Informatics, 34, 1-16.

Kumar, P. (2012). Road features extraction using terrestrial mobile laser scanning system. National University of Ireland Maynooth, http://eprints.maynoothuniversity.ie/3995, 300 p., 25-January-2015.

Kumar, P., McElhinney, C. P., Lewis, P. and McCarthy, T. (2013). An automated algorithm for extracting road edges from terrestrial mobile LiDAR data. ISPRS Journal of Photogrammetry and Remote Sensing, 85, pp. 44-55.

Kumar, P., McElhinney, C. P., Lewis, P. and McCarthy, T. (2014). Automated road markings extraction from mobile laser scanning data. International Journal of Applied Earth Observation and Geoinformation, 32, pp. 125-137.

Kumar, P., Lewis, P., McElhinney, C. P. and Rahman, A. A. (2015). An algorithm for automated estimation of road roughness from mobile laser scanning data. The Photogrammetric Record, 30 (149), pp. 1-16.

Louhaichi, M., Borman, M. M., \& Johnson, D. E. (2001). Spatially located platform and aerial photography for documentation of grazing impacts on wheat. Geocarto International, 16(1), 65-70.

Mancini, A., Frontoni, E. and Zingaretti, P. (2009). Automatic extraction of urban objects from multi-source aerial data. International Archives of Photogrammetry, Remote Sensing and Spatial Information Sciences, 38 (3/W4), pp. 13-18.

Mumtaz, S. and Mooney, K. (2009). A semi-automatic approach to object extraction from a combination of image and laser data, International Archives of Photogrammetry, Remote Sensing and Spatial Information Sciences, 38(3/W4), pp. 53-58. 
Musialski, P., Wonka, P., Aliaga, D. G., Wimmer, M., Gool, L. V., \& Purgathofer, W. (2013, September). A survey of urban reconstruction. In Computer graphics forum (Vol. 32, No. 6, pp. 146-177).

Oda, K., Takano, T., Doihara, T. and Shibasaki, R. (2004). Automatic building extraction and $3 \mathrm{D}$ city modeling from LiDAR data based on Hough transformation. International Archives of Photogrammetry, Remote Sensing and Spatial Information Sciences, 35 (B3), pp. 277-280.

$\mathrm{Pu}$, S. and Vosselman, G (2006). Automatic extraction of building features from terrestrial laser scanning. International Archives of Photogrammetry, Remote Sensing and Spatial Information Sciences, 36(5), pp. 25-27.

Rutzinger, M., Elberink, S. J. O., Pu, S. and Vosselman, G. (2009). Automatic extraction of vertical walls from mobile and airborne laser scanning data. International Archives of Photogrammetry, Remote Sensing and Spatial Information Sciences, 38 (W8), pp. 7-11.

Schnabel, R., Wahl, R., \& Klein, R. (2007, June). Efficient RANSAC for point- cloud shape detection. In Computer graphics forum (Vol. 26, No. 2, pp. 214-226). Blackwell Publishing Ltd.

Tucker, Compton J. "Red and photographic infrared linear combinations for monitoring vegetation." Remote sensing of Environment 8.2 (1979): 127-150.

Vosselman, G. (2009). Advanced point cloud processing, Proc. Photogrammetric Week, Stuttgart, 7-11 September, pp. 137-146.

Revised August 2019 